\title{
Adolescents with anxiety and depression: is social recovery relevant?
}

\author{
Abstract \\ Social recovery has become a prominent aspect of mental health service design \\ and delivery in the past decade. Much of the literature on social recovery is derived \\ from first-person accounts or primary research with adult service users experiencing \\ severe mental illness. There is a lack of both theoretical and empirical work which \\ could inform consideration of how the concept of social recovery might apply to \\ adolescents experiencing common (non-psychotic) mental health problems such as \\ anxiety and depression. The current study was conducted to understand the process of \\ experiencing anxiety and depression in young people. Semi-structured interviews were \\ conducted with nine adolescents with anxiety and depression (seven girls and two boys \\ aged 14 to 16) and twelve mothers who were recruited from a specialist Child and \\ Adolescent Mental Health Service in the South of England. Thematic analysis indicated \\ that young people do experience a process of 'recovery'; the processes participants \\ described have some congruence with the earlier stages of adult recovery models \\ involving biographical disruption and the development of new meanings, in this case of \\ anxiety or depression, and changes in sense of identity. The accounts diverge with \\ regard to later stages of adult models involving the development of hope and \\ responsibility. The findings suggest that services should attend to social isolation and \\ emphasise support for positive aspirations for future selves whilst also attending to \\ young people's and parents' expectations about change. Methodological challenges face \\ enquiry about 'recovery' given its connotations with cure in everyday language.
}

Keywords: UK; social recovery, adolescent; mental health; families; qualitative 


\section{Introduction}

In the past decade, recovery-oriented service provision has become a prominent concept in international mental health policy (particularly the USA, Australia, New Zealand and the UK) and, indeed, now serves as a standard for mental health service reform (Pilgrim, 2008). Little attention, however, has been paid to the application of the recovery agenda to mental health services for young people. The meaning and relevance of recovery to young people and their caregivers has largely not been explored and it is not clear whether or how models based on adult service users have relevance to younger people. The current study is the first to attempt to explore the concept of social recovery for young people using a bottom-up, data-driven, methodology. We argue in this paper is that it is inappropriate to extrapolate recoveryoriented practice for young people from adult recovery frameworks. We also question the extent to which recovery processes can be distinguished within the context of cognitive and social development typical of the late childhood and adolescent period and discuss the implications for how recovery-oriented practice might be formulated.

Within the UK mental-health context, there is an expanding literature on recovery that incorporates a diverse range of perspectives. Publications include service user accounts, conceptual reviews, policy documents, position papers, and a modest number of empirical studies. Within this literature, several authors have pointed out the potential for confusion in the meaning of this term for both mental health professionals and users of their services. Fundamental debates concern the nature of recovery whether it is a process, an outcome, or both - and the meaning of recovery. For some, recovery might stand for symptom amelioration - a process of becoming healthy once again. For others, recovery might mean living a meaningful life despite the continued 
presence of symptoms (Davidson \& Roe, 2007). The latter has been referred to as 'social recovery' and it is with this construction that the current paper is concerned.

Social recovery in mental health has its origins in the service user or 'survivor' movement, giving it a distinctly socio-political emphasis. First-person narratives of mental health problems contributed to the idea that social recovery involved overcoming traumatic experiences related to having a mental illness label. This 'recovery from invalidation' is linked to experiences of stigma, social disadvantage, and loss of identity as well as direct experiences related to the mental health system (Pilgrim, 2008). This notion of social recovery also grew out of observations that although a significant proportion of individuals with severe mental health problems were able to achieve complete symptomatic recovery, other individuals were able to live meaningful lives in the presence of symptoms (Davidson \& Roe, 2007). Despite the idiographic emphasis inherent within social recovery, conceptualizations of it within the literature appear to cohere around a small number of themes which include empowerment and control, positive identity, connectedness, hope, optimism, and discovering meaning and purpose (Tew et al., 2012). It seems, therefore, that although recovery may be unique to the individual, empirical work and personal accounts suggest common features and processes. This may not be altogether surprising given that people are likely to share broad motivations around personal control, social engagement, and developing/maintaining a purposeful life. However, these common features and processes are derived from the accounts of adults.

In addition, the social recovery literature predominantly focuses upon severe mental illnesses, primarily those individuals diagnosed with schizophrenia, rather than common mental health problems, such as anxiety and depression. For example, in their 
review of the British literature on 'recovery and mental health', Bonney and Stickley (2008) used 'recovery', 'schizophrenia' and 'psychosis' as search terms. In later reviews of both the British peer-reviewed (Stickley \& Wright, 2010a) and 'grey' literatures (Stickley \& Wright, 2010b) the focus was exclusively on adult mental health. Although a recent study on recovery in anxiety and depression has been reported by McEvoy, Schauman, Mansell and Morris (2012), their analysis of the experience of 98 adult service users who had received a brief primary care intervention was focused on recovery from anxiety and depression rather than recovery in the presence of symptoms.

It follows that there is a question about the extent to which current conceptualizations of mental health recovery that are almost exclusively derived from severe and enduring mental health problems might be applied across the board, and further, an equally problematic question is the extent to which adult models can apply to young people. Adult recovery conceptualizations imply that the individual requires the ability to integrate potentially opposing self-concepts; particularly, the idea of developing meaning and purpose in the face of continued psychological and functional difficulties. As Harter (2003) observes, young people as old as 16 have difficulty in integrating contradictory self-concepts and that awareness of opposites promotes confusion and distress. Furthermore, Harter notes that the development of a coherent self-concept in adolescence is a particularly challenging task given the central importance of the evaluation of others during this period of development. For this reason it seems indefensible to base a concept of social recovery for young people on adult models without prior empirical testing of its applicability.

Another issue confronting investigators of social recovery is methodological: if we are to develop data-driven conceptualizations of recovery, how might we access a 
construct with which participants may not be familiar and without imposing pre-existing assumptions onto them? In their qualitative study of recovery from schizophrenia in adults, Noiseaux and Riccard (2008) asked informants to describe their perceptions of 'recovery' and what facilitated or impeded this. Clearly, this requires an ability on the part of participants to engage with the idea of social recovery and that certain factors can enhance or prevent it. In one study considering the applicability of adult models to young people, Friesen (2007) adopted a top-down approach to exploring recovery with service-providers, children and their parents in the United States by providing definitions of the concept. Although participants agreed with some aspects of the definitions it remains an empirical question as to whether their self-constructed ideas of recovery would be consistent with the conceptualizations given to them. Nevertheless, Friesen's study gives some insight into the potential problems associated with the application of recovery to mental health services for young people. A significant concern raised by participants in Friesen's study was that the term 'recovery' is potentially misleading, due to its inference of cure in everyday vernacular, and inappropriate from a developmental perspective since it suggests recovery from illhealth rather than the promotion of social and emotional wellbeing that is the common emphasis in mental health services for young people.

In sum, given the absence of theoretical and empirical work on recovery in young people with common mental health problems, it is unclear how current conceptualizations of social recovery are relevant to this group and how developmental considerations shape the meaning and applicability of the recovery concept. These questions are important given the emphasis in UK mental health service policy that recovery should apply across the age and symptom spectrum. The current study was 
designed to address the following question: what accounts do young people and their parents construct when asked to talk of their experiences from onset of symptoms, through to engagement with mental health services, and subsequently? Our aim was to assess if the accounts produced could meaningfully contribute to the question of the relevance and nature of recovery for young people with common mental health problems and their parents without organizing those accounts around pre-existing frameworks. Eliciting personal experiences is congruent with the philosophy of recovery given its emphasis on personal meaning.

\section{Methods}

Recruitment

Ethical approval was granted by a UK National Health Service (NHS) Research Ethics Committee. A service-user research advisory group was formed comprising four young people aged under 16 years who had experienced NHS mental health treatment. The four advisors were consulted on study materials, interview questions and distress management during interviews. All were paid for their time. Data were collected in the first six months of 2010. Participants were recruited from a specialist Child and Adolescent Mental Health Service (CAMHS) in Southern England. The target group was young people with a primary diagnosis of anxiety or depression who were currently, or had previously been, treated within CAMHS when they were aged between 10 and 16 years, and their parents. Identification of suitable families was made by the consultant clinical psychologist in the CAMHS team. One hundred and fifty eligible families were invited by letter to participate. Nine young people ( $6 \%$ of those invited) 
aged between 14 and 16 were included in the study, seven girls and two boys. All were White British and living in the family home. All but one was currently participating in formal education. Five were currently being treated in CAMHS and four had been discharged. Seven had been considered by CAMHS professionals to meet diagnostic criteria for anxiety disorder (2 Obsessive-Compulsive Disorder, 1 Panic Disorder, 3 Generalized Anxiety Disorder) and three had been considered to meet diagnostic criteria for depression. Twelve White British mothers ( $8 \%$ of those invited), aged between 35 and 54, took part. Nine were employed in professional or managerial roles, two were service workers, and one was unemployed. Their children (ten girls, two boys) were aged between thirteen and seventeen. Eight of the children were still in CAMHS and four had been discharged. The children of seven of these mothers were interviewed in this study.

\section{Data Collection}

All families received an invitation letter attached to which was an information sheet and consent form for both the participating parent and the child. The invitation letter specified that only one parent per family should take part. Parents were asked to provide written consent for children under 16 years old to take part. Mothers and all young people provided written consent for their own participation. Once signed consent forms were received, interviews were arranged. Prior to the interview beginning, all participants were advised that they could terminate the interview or not answer particular questions without consequence. Breaks were provided where needed. Interviews were conducted face-to-face either at the CAMHS clinic or in the participant's home. A semi-structured interview schedule was developed and refined 
based on consultation with the advisory group. In developing the interview schedule, the term 'recovery' was not used initially due to reasons outlined in the Introduction. It might have been meaningless at best, or confusing and antagonistic at worst, for young people and their parents. Therefore, young people were advised that the interview was concerned with understanding their experience of anxiety/depression. In order to access accounts of experience over time, questions focused on (1) the period when the young person first started to experience problems; (2) when they first went to CAMHS; (3) the current time; (4) what they think has changed between these time points; (5) how they think any changes had come about; and, (6) how they envisaged the future. Similarly, mothers were advised that the interview was focused on how they have understood their child's experiences over time using similar question areas as described for young people. Following these questions, the idea of social recovery was described to the participants and they were asked their thoughts on the description and its meaning in the context of their own experiences. Each interview lasted between 30 to 60 minutes and was digitally recorded. At the end of the interview, participants were given the opportunity to ask questions. All participants were sent a summary of the study findings.

\section{Data Analysis}

Interviews were transcribed verbatim and anonymized. Thematic analysis was used to analyze the interview transcripts within a contextualist epistemological framework. A contextualist stance recognizes that the interview occurs within a specific historical and socio-cultural context and, therefore, meaning derived from data can be understood only within the context of its production and analysis. This position 
is consistent with the idea that the meaning of recovery is a product of historical, social and cultural context and not an invariant truth waiting to be discovered. Additionally, an interview is a contrived situation with a range of demand characteristics on both respondent and interviewer. An inductive and interpretative approach was applied to the data given the aim was to develop an understanding of the experiences of young people and their parents rather than to fit pre-existing ideas to the data. Interpretations are grounded in the report by way of presenting multiple examples of participants' speech. Analysis proceeded following the stages outlined by Braun and Clarke (2006). Separate researchers coded the interviews with young people and mothers. Each began by familiarizing themselves with the set of transcripts. Initial coding of interesting features of the data was followed by the collation of codes into potential themes. Themes were then reviewed against the transcripts and an initial thematic map was produced. Themes were then refined, defined and renamed following further engagement with the data. A final thematic map for each set of interviews was drawn up. Throughout this process, a second researcher acted as an 'auditor' to this process. The purpose of audit was to question correspondence between interpretations, themes and the interview data. Following completion of the two individual analyses, a third analyst then assessed thematic correspondence across these two separate analyses in order to derive overall themes and subthemes. Main themes between the two separate analyses showed a high degree of similarity. An 'audit' of this process was then made by a further analyst assessing the coding tables of the two individual analyses against the thematic map of the integrated analysis.

\section{Findings}


In this section, the three analytic themes developed following integration of the separate analyses of interviews with young people and mothers are presented. Verbatim extracts from participants' transcripts are presented throughout; however, ellipses (...) have been used to indicate where material has been omitted for succinctness. Ellipses are used only where the omission does not alter the interpretation of the text.

Participants have been given pseudonyms. The data yielded from asking participants about the definition of recovery were not included in the analysis because young people seemed merely to acquiesce with these ideas or be unable to think about them. The data from mothers on this question did not add appreciably to the analytic themes but some noteworthy aspects of their responses are considered in the Discussion section of this paper.

Theme 1: Loss of Self

Whilst their initial experiences are somewhat varied, the young people's accounts reflect a common theme pertaining to relational transformation resulting in a lost self. Relational transformation reflects the challenge to the young person's selfconcept from two sources - through a disruption in how they relate to themselves (i.e. understand and experience themselves); and, in how they relate to others. This theme reflects the idea that identity is socially embedded as it encompasses an intra-psychic dimension that interacts with an interpersonal dimension. Overall, the loss of the self comprises loss of psychological, social and relational integrity.

Loss of the self involves an intra-psychic transformation that the young person does not understand and, in some cases, finds extremely frightening. Most obviously, 
this loss of self relates to the experience of powerful and inexplicable thoughts, feelings, and behaviours:

"Well I had sort of a, a feeling of like fear inside me that I was, well something's like just I don't know what but something like I don't know I was going to get beaten up or something like that and it was like really scary" (Callum).

The intensity of fear impacts on the young person's ability to manage what they are experiencing: "I'd just freeze with anxiety, I was not able to cope" (Zoe). At this stage it is understandable that young people indicate they do not know what is wrong with them, further undermining their self-coherence. Justine's statement indicates her complete incomprehension about what was, and is, going on: “I don't know. I don't know really. I just don't know. I don't know, I find it's like even like a really little thing, like I don't know, I just I don't know”.

For some there was ambivalence about the self, a sense of not being sure what the essential problem was. For example, Anna seems to fluctuate in her self-evaluation giving a strong sense of personal uncertainty: “I didn't like who I was. I don't know if, I don't know. I was ok with who I was but I was just really unhappy”.

Mothers' accounts supported this sense of incomprehension and feeling lost: "And we would talk to him and he would just say, 'I don't know why it is'” (Joanne). In the presence of powerful and often frightening feelings and sensations coupled with a lack of understanding of what is wrong with them, young people commonly experienced erosion in their confidence. Phoebe indicates the frustration involved: "I wasn't very confident at all. Umm, and I sort of really wanted to be more confident but you know you just can't bring yourself to quite do it". 
In the context of powerful and inexplicable thoughts and feelings and an erosion of confidence, young people describe a diminishing interpersonal and social world, something that was reported commonly by the mothers interviewed. There was a sense in which they had lost their place in the world. For some young people, there are initial fears that they will not fit in, perceiving themselves to be vulnerable to the judgement of others. Withdrawal may then ensue and this may manifest in actual or desired avoidance of school participation. It may also manifest in avoiding interaction with others. Sometimes this is due to specific symptom-based difficulties. For example, Charlotte would not stay overnight with friends as this meant she would not be able to engage in the rituals she needed to do. At other times, avoidance was related to the intolerability of being around others when one is anxious. Sometimes, the avoidance is pervasive:

“I just couldn't bear myself to be around people...I would stay in the house all day full stop but if I had to be around my sister and my mum, god forbid, so eventually I just stopped sleeping at like night, and started sleeping in the day just to avoid them" (Zoe).

Young people described restricting activities they used to enjoy due to the problems they were experiencing. Most commonly, young people described not joining in with their peer group or cancelling social arrangements, further emphasising their loss of place:

"It was hard because like all my friends around me was going out and doing things and I just sort of felt left out but actually it was my own fault for not going out and saying no" (Maya).

One way in which loss of place might be experienced is by the young person finding themselves in a location that they would never previously have imagined. 
David indicated that going to a mental health service was something he had never before conceived of as personally-relevant:

"I didn't think I'd ever have to go to a place like that. Umm, but I know that it had to be done sort of thing... It was just the people and just the place and just me being in a place like that".

In the face of multiple losses and uncertainties, young people commonly expressed a desire to be 'gone' from their lives. This was commonly articulated in language of wanting to die or to harm oneself. For some mothers, the young person's disclosure of wanting to die marked the start of their awareness of the problems. Most young people indicated that suicide was never a possibility and, as Maya's statement indicates, this might be vocalised as a way of expressing the intensity of their distress: "Sometimes I used to say to my mum 'I'm gonna tie this rope up the curtain, on a curtain and jump off of it' but I never did do it, I just sort of thought about it but never did get that close”.

The diminution of the young person's interpersonal and social world serves to constrain possibilities for current and future thought and action and enhances the perceived contrast between the young person and others, further amplifying a sense of the lost self and a loss of connection with others. Most obviously, restricted connection comes about by way of the young person failing to disclose their problems and fears, either fully or in part. For example, Phoebe describes always trying to act 'normal' with her peer group. The desire to prevent others from knowing means concealing aspects of the self that have become significant for the young person but that are perceived to be part of an unacceptable identity: “And I was like really frightened of seeing someone 
that I knew and them seeing me like that because I was so embarrassed about it" (Emily).

Emily is making an assumption that other young people would not identify with her experiences and thus indicates a discrepancy between her and the norm group. Consequently, losing connection with others comes to pass not only in the effects of being depressed and anxious but in self-other comparison processes. To the extent that self-concept is socially-embedded, social comparisons serve to produce a disconnection from others as one appraises oneself as 'different to', with its attendant implications for self-evaluation. Hence, not only do these comparisons provide a source of meaning, as realising the self as 'different', they also serve to reinforce the separation from others, whether perceived or actual:

"You sort of see other people, sort of not being sort of freaked out and anxious or depressed whatever, it's, you sort of feel like 'why can't I be more like that?'”(Phoebe).

A corollary of assuming that others do not share ones experience is the perception that those others would be incapable of understanding ones distress. This seems to be based on the notion that one must experience in order to empathise. For example, Emily makes the dual assumption that there is no-one else her age that experiences what she does and, therefore, that there is no-one to whom she can relate. Callum expresses a preference for being called a 'wuss' for repeatedly declining his friends' invitations for adventure for fear of the consequences of disclosing: "I couldn't say anything because it would make it worse”. The assumption of a lack of empathic understanding promotes distance between young people and their peers. Young people may, therefore, choose to keep their distance from others which results in the loss of friends and a sense of being alone: 
"you feel quite alone when you're growing up with like things like that because there's not really anyone who understands it" (Zoe).

Most young people described their willingness and ability to discuss their problems with their mother, something that had not changed following onset of problems. A few, however, described a period of sometimes protracted concealment that progressively, and reluctantly, gave way: "but when it started getting serious and obviously I couldn't help trying to keep it in but it just leaked out all the time" (David).

Mothers' accounts also reveal the assumption that lack of experience relates to lack of empathic understanding: "It was difficult because she looked at me being unsympathetic 'cause I didn't understand and didn't know what to do”. (Samantha). On occasion, mothers did express the challenge to empathic understanding: "Having to actually stand back, distance myself and say no, she's quite genuinely not dealing with this. It seems like a complete load of rubbish but it's real" (Patricia).

\section{Theme 2: Renegotiating the self}

Just as loss of the self comes about by way of disruption in how one understands and experiences oneself, both intra-psychically and in relation to others, then the process of re-authoring the self occurs by way of dynamic intrapersonal and interpersonal processes. The accounts suggest that these young people are engaged in a process of renegotiating their self-concept rather than regaining a lost, prior self. However, what is evident in these accounts is a significant degree of ambivalence and uncertainty in this process. The instability in the accounts may indicate young people who are actively engaged in the process of reclaiming themselves. Alternatively, it may reflect the essence of renegotiation as incorporating both progressive and regressive 
elements. As such, young people simultaneously talk of themselves as progressive agentic beings whilst simultaneously rejecting ideas around agency and progress.

When describing the onset of their problems and the quality of life at that time, many young people talk of discomfort and challenge, particularly in peer relationships. These issues, coupled with the anxiety and depression they were experiencing, produced pervasive feelings of low self-esteem and self-efficacy, lack of confidence and motivation, and hopelessness. Opening up to others was central for some young people in them beginning to tackle their difficulties, although this was almost exclusively to family and service professionals rather than peer group members. For example, Callum linked improvement definitively with being able to talk to a service professional: " $b u t$ the talking kind of things which is here has made a like big impact and has stopped the illness".

Although Charlotte feels her symptoms have not really changed, her ability to be more open has: “I guess there's been change in, there hasn't been a lot of change in myself and my OCD... but there's been a change in how I can approach to talk about it with someone and feel a lot more comfortable”.

Some mothers also reported an increased willingness in their children to discuss their concerns. In contrast, some young people expressed ambivalence about the value of talking, perhaps revealing their expectations of what it is capable of achieving. For example, Justine states that talking to her mother is "helpful but like I don't know, it still, it still doesn't make it like better, like I still like end up just crying or whatever like that".

Some young people perceived that they were re-engaging with others socially and recognising that they were able to do things that they would previously have found 
difficult. Maya notes how this now means she does not feel so different when she compares herself to others her age. However, David's response suggests he still perceives a difference from others:

David: "All moving on with their lives and you're just slowly moving backwards". Interviewer: "Does it still feel like that now?"

David: "Yeah, it does yeah"

The accounts of some young people indicated a growing realisation of strengths and personal resilience. With the recognition that the young person has dealt with adversity comes a renewed sense of confidence. These young people have proved to themselves that they can do things that they did not think possible or things they perceive that young people their own age would not be capable of. Additionally, this sense of resilience endures despite the continued presence of difficulties: “Just like even though I find, like let's say going into [town] it's difficult, I can still do it. It's really difficult... I still have difficulties with it but I can still do it. It's like, I really kind of get that" (Emily).

Some young people also talked of pushing themselves on a daily basis to face their fears and deal with their problems. Their accounts gave a sense of fortitude and a realisation of how they had changed in this respect: "I don't get embarrassed like if I did something wrong. I just get up and try again. Whereas before I wouldn't” (Anna).

In some accounts, there was a sense of self-belief and determination. Mothers also identified increased resilience in their children and a willingness to be more proactive: "She can cope with a lot more. It's just when stressors come at the same time she can't cope....now it will take a lot of things to get her" (Jill). 
In contrast, Justine was unable to claim any sense of agency in coping with her problems indicating a belief that change had occurred without any conscious input on her part: "I just found I was doing it". Other young people were not able to express an understanding of change. For example, Zoe indicated that much had changed but that “it's impossible to tell what it is though". Emily thought because change had taken place over a long time it was difficult to recognise what was different. The young people often struggled with school participation or academic work. Mothers discussed realising and valuing other strengths in their children and hoped that this might be a way towards renewed confidence.

In renegotiating their self, a few young people describe the importance of service professionals in validating their experiences and helping them to begin to move on from them. Mothers thought that knowing what 'it' was helped their child regain control and enabled them to understand triggers and unhelpful thinking. Maya describes how service engagement helped her to realise that not everyone judges her negatively and, consequently, she was able to re-engage socially. A few young people also presented a more accepting view of their problems and a motivation to work with them, as noted by a mother: "rather than 'I hate it, I hate it, I hate it, make it go away' acute loathing of it, 'ok I am like this what do I do?'” (Patricia).

Young people who described more self-accepting attitudes also talked about a sense of common humanity. This entailed a realisation that they are likely no different to others. Importantly, this may reduce social comparison processes that serve to distance them from others. Additionally, some talked of an increased sense of understanding and empathy towards others in a similar situation: "I think it helped me to be more understanding of people who are feeling low or something. It's really 
helped me in that kind of aspect so like I'm quite good at talking to people when they're the low mood who a lot of my friends don't understand how to talk to somebody" (Zoe). This may also give young people a sense of increased confidence and a place in the world.

However, within this theme was evidence of contradiction, ambivalence and uncertainty. For example, despite thinking he has a better understanding of his problems, David goes on to state: “I just hate it. So I can't cope with it any better really so no, I don't really think anything's really changed to be quite honest”. Similarly, Zoe's comments about the development of empathic understanding seem to contrast with her somewhat nihilistic appraisal of her own agency: “there's just nothing around you and you can't kill yourself, can't do anything, nothing will take it away so it's just like the worse feeling in the world yet there's nothing you can do about it".

Although most mothers thought that their child had made progress in managing their problems, some mothers expressed the view that nothing had changed. However, they then went on to report improvements in their child's functioning and/or understanding. Contradiction in the accounts of young people and mothers might be underpinned by fluid expectations about the type and magnitude of change that should be achieved.

\section{Theme 3: Anticipation of future self}

Most of the young people were able to state an immediate goal such as finishing their exams, going to college or university, learning to drive. They also stated aspirations such as earning good money, maintaining intimate relationships, and being happy and successful. However, when thinking about their current problems and the 
future, few young people were able or willing to engage with this conceptually and, of those that could, a common hope was to be symptom free.

David and Callum expressed the belief that they will one day 'get over' their current difficulties. Both drew on therapeutic ideas of breaking the vicious cycle of anxiety by facing their fears. It is evident that their conceptualisation of dealing with their problems means symptom eradication. Callum further explains his belief that symptom eradication will come about by a process of maturation, thus revealing ideas about his own agency in this process.

"I think when I get older I will eventually find myself being able to go out on my own...basically this whole anxiety is stopping me from having like a good time because it's stopped me from going out...soon as I get older and the anxiety stops then I'll be fine”.

His use of the term 'eventually find myself being able to' indicates Callum's belief that time will be sufficient to alleviate his problems. Similarly, Phoebe expressed the belief that maturing had facilitated the changes she had experienced. In neither account is there an expression of how maturation provides a means for change. Instead, there is an implicit belief that agency and, by implication improvement, is connected with growing older.

Most young people expressed the hope that their problems would stop. Charlotte's and Emily's accounts revealed that, although they had found talking to a service professional helpful, they had an expectation that intervention would stop their 
problems: “but it's a shame that I didn't come away from it and... and stop OCD altogether and I hope one day I will" (Charlotte).

Callum wanted his problems to 'disappear' so that he could lead a 'normal life'. David pointed out it was 'obvious' that the best result would be not having it any longer. When considering what the goal of recovery might be, Zoe's perspective corresponds with the views of other young people that eradication should be the aim: "Like, if it's someone who can theoretically get over everything, their recovery should be to get over everything and not settle for less, whereas with people who can't, probably it'd be impossible to sort of get past certain things, then they can settle for that as recovery”.

Despite hopes for problem eradication, the accounts of a number of young people expressed ambivalence in this regard. Phoebe expressed uncertainty about wanting to be 'like everyone else'. Callum and Zoe expressed a fear that they would be unsafe if they gained independence and freedom from their problems.

All mothers wanted their children to no longer experience anxiety or depression. However, the majority felt that it might be present in their child's lives in the future. In this respect, they expressed hope that their child would be able to manage it, have an independent life with it, and be happy. However, there was a great deal of uncertainty and worry expressed about their child's ability to cope as an adult. Only one mother felt that their child's experience was a stage in her growing up and would not return. This mother saw her daughter's difficulties as being due to her temperament and that growing up and appreciating life more would lead to improvements. Another mother, although not thinking that her child would grow out of low mood/worry hoped that, 
with changes in hormones through growing older, things would improve. It is very possible that maternal ideas about distress and maturation have been transmitted to young people and come to form part of their worldview.

\section{Discussion}

Broadly, the analysis of accounts of the experience of anxiety and depression and service engagement presented here suggests a process of loss and rediscovery. At this analytic level, the experiences of young people are similar to accounts in the adult recovery literature (e.g. Young \& Ensing's, 1999, qualitative study of 18 adults with severe psychiatric disorder; Spaniol et al.'s, 2002, qualitative study of 12 adults with schizophrenia; Andresen et al.'s, 2003, thematic analysis of 28 published personal accounts of recovery from schizophrenia/severe mental illness, 10 'consumer' articles on recovery, and 8 qualitative studies). The theme 'loss of self' represents the experience of biographical disruption through inexplicable and frightening thoughts and feelings. Withdrawal follows either due to the nature of the problems or because of embarrassment and fear of negative evaluation, or both. As such, a spoiled identity (Goffman, 1968) develops. There is a heightened perception of self-other difference and what follows is isolation which is compounded by a belief that others cannot understand. This description has conceptual similarity with Andresen et al.'s (2003) 'moratorium' (a period of hopelessness, identity confusion and social withdrawal) and Spaniol et al.'s (2002) 'overwhelmed by the disability' (a period of fearfulness, confusion and disconnection from self and others). The theme 'renegotiating the self' represents a period of greater awareness of and engagement with the problem, the main catalyst for which is engagement with services. During this period there is growing 
awareness of strengths and resilience and some reduction in perceptions of self-other distance, resulting in social re-engagement. However, this is not representative of the full sample and there is evidence of ambivalence regarding the perception of change in some accounts. This theme has some conceptual similarity with Andresen et al.'s (2003) 'awareness' - a period during which the person realises a different self is possible - and Spaniol et al.'s (2002) 'struggling with the disability' - a period of increasing knowledge of the problem, some engagement with it and growing selfconfidence but with some fear of becoming destabilised. The fear of destabilisation might be evidenced in young people's accounts by the presence of ambivalence although this is purely speculative. Destabilisation fears might also account for the reluctance of those parents who declined participation in this study. The theme 'anticipation of future self' is the thinnest and mostly comprises hope for symptom eradication. At this point the data depart from adult recovery conceptualizations. Andresen et al.'s and Spaniol et al.'s conceptualizations involve periods of active engagement with recovery, taking responsibility and control, being resilient, positive and forward looking. Spaniol et al.'s final stage of 'living beyond disability' is not evidence-based but instead is derived from descriptions in the self-help literature. It is not to say that the current data do not contain elements of resilience and positivity but the evidence for these as distinct and universal qualities of the experience is not sufficiently compelling. The ability to look towards the future was notably absent in the young people's accounts. It was not that young people imagined a less positive future with symptoms; instead, they found it difficult to conceptualize a future like this. This might reflect sampling bias or be due to a limited sample size. However, the literature suggests some possible alternative explanations. For example, in a study of 935 
adolescents and adults assessing behavioural and self-report measures of future orientation Steinberg et al. (2009) found that adolescents under 16 showed a consistently weaker future orientation than those participants aged 16 or over. Second, Seginer and Lilach (2004) point out that loneliness is prevalent in adolescence. Findings of their study indicate that lonely adolescents score lower than more sociallyembedded adolescents on relational future orientation (i.e. social relationships, marriage and family) but not instrumental future orientation (i.e. education and work). Although Seginer and Lilach's study focussed on non-clinical $11^{\text {th }}$ grade Israeli adolescents who generally exhibited mild levels of loneliness, this study suggests some caution in interpreting reduced future thinking purely in relation to common mental health problems. That is, low future-orientation may be prevalent due to loneliness per se rather than being an indicator of psychological disorder. It may also be that young people are not at the stage in which they have reached the ability to reflect on and imagine possible future selves. If so, this has important implications for how recoveryoriented practice is implemented in services for young people, suggesting that services should attend to social isolation and emphasise support for positive aspirations for future selves. As noted, however, this might be a challenging task for adolescents who might not have developed the ability to integrate opposing self-concepts and who might find this confusing or distressing (Harter, 2003).

Andresen et al.'s (2003) formulation, as well as the general literature on recovery, emphasises the individual assumption of responsibility. A significant contrast here comes from the interviews with mothers when asked about the definition of recovery. The common view was that young people cannot be expected to take responsibility and should be allowed to have the expectation of a symptom-free future. 
The accounts reveal expectations from all respondents about the ways that young people should be living their lives. As such they reveal cultural representations of the normal roles, abilities and expectations of young people in society - there is a 'normal' life that the young person is missing out on and should be restored to.

Overall, the data in the current study present a mixed pattern of correspondence with the adult recovery literature. The limitations of the current study notwithstanding, it might be proposed that differences emerge partly because adolescence is a stage of significant identity transition and that young people are more dependent on systems of care than adults given their developing emotional, cognitive and social skills. Jacobson and Greenley (2001) proposed that the interaction between intra-psychic conditions and external conditions produces the recovery process of an individual. As such, theorizing recovery-enhancing factors in this group needs to be contextualized within developmental processes such as attachment, physical and cognitive maturation, and social identity development. It also needs to involve the construction of meaning within family systems, such as the understanding of psychological distress in the context of adolescent development and maturation, and services that support young people. Finally, a distinction needs to be made between understanding intrapersonal and interpersonal expectations and processes of change on the one hand, and defining recovery-oriented services for young people and their carers on the other. The social recovery movement has driven State level consideration as to what constitutes meaningful progress for service users and their families and what the goals of intervention should be. However, it is unlikely that one approach to recovery will be acceptable to all. In this study, although engaged in the process of living with their problems, the young consumers and their mothers hope for clinical recovery. 
Furthermore, as Pilgrim (2008) notes, psychiatric services cater not only for those with chronic psychosis but also for "those who are sad and frightened" (p302) and, therefore, the specifics of recovery will vary. Future research and theorizing needs to attend to the potential variability in 'recovery-oriented' views and practices in specialist mental health services for young people if we are to understand the challenges in achieving it and recovery-oriented services ought, therefore, to consider including explicit consideration of expectations for the future for both parent and child.

At this point, the methodological limitations of this study should be examined. The uptake rate was poor (6\% of young people and $8 \%$ of mothers). Reasons for nonparticipation included reluctance to talk, fear of jeopardizing progress, life demands and service dissatisfaction. Parents were required to consent to their child's participation. Understandably, if parents are concerned about destabilization then they will be less likely to consent to their child's participation. However, this might result in young people who would be willing and able to participate being excluded and the resultant sample being biased. Additionally, parents may fear reactivating their own distress. This raises the question of whether the sample is biased in favour of those parents who considered their families more emotionally and cognitively able to deal with the demands of the interview. Our aim had been to sample young people who were at different points in their engagement with services so as to avoid a sample comprised purely of respondents who had been discharged and whose stories might reflect a more progressive tone. However, recruiting young people who are currently in services may be difficult due to the destabilization fears discussed above. In order to develop findings based on a larger sample, it may be necessary for future studies to focus on older, discharged children. Further limitations of the sample are that no fathers agreed 
to participate and all respondents were White British. It remains to be seen how the findings might apply to other caregivers and different ethnic groups where the interpretation and negotiation of distress might differ from a White British sample. The current study also only focuses on young people with anxiety and depression and future work is required that focuses on other mental health problems.

The final critical question is - given the methodology employed, to what extent can these findings claim to be relevant to the understanding of social recovery? In order to make such a claim, some assumptions must be acknowledged. The first is that recovery is a discernible process which, although non-linear, involves a number of discrete but inter-related phases that may be described. Adult recovery models are partly based on such an assumption. The second assumption is that the process of recovery involves change - not purely in symptoms, although this may well happen, but more crucially in sense of self. These working assumptions led us to develop an interview which enquired about experience over time and which focussed on personal change. Although in this paper we claim this has relevance for understanding recovery, we necessarily acknowledge that these processes might be labelled in other ways - for example, as coping, adaptation, accommodation and resilience and may reflect processes of maturation rather than processes of 'recovery' per se. This significant conceptual issue has not, however, been resolved in the adult recovery literature. The findings of the current study do, however, question the applicability of adult models to young people and underscore the lack of research in this age group despite State policies on recovery-oriented practice across the developmental spectrum. More critical debate and robust empirical research is needed to address the question of whether and how the 
concept of social recovery adds value to service design and delivery for young people and their families.

\section{References}

Andresen, R., Oades, L. \& Caputi, P. (2003). The experience of recovery from schizophrenia: towards an empirically validated stage model. Australian and New Zealand Journal of Psychiatry, 37, 586-594.

Bonney, S. \& Stickley, T. (2008). Recovery and mental health: A review of the British literature. Journal of Psychiatric and Mental Health Nursing, 15, 140 - 153.

Braun, V., \& Clarke, V. (2006). Using thematic analysis in psychology. Qualitative Research in Psychology, 3, 77-101.

Davidson, L., \& Roe, D. (2007). Recovery from versus recovery in serious mental illness: One strategy for lessening confusion plaguing recovery. Journal of Mental Health, 16, 459-470. DOI: 10.1080/09638230701482394

Friesen, B.J. (2007). Recovery and resilience in children's mental health: views from the field. Psychiatric Rehabilitation Journal, 31, 38-48. DOI:10.2975/31.1.2007.38.48 Goffman, E. (1968) Stigma - Notes on the Management of Spoiled Identity, Reprinted 1990. London: Penguin.

Harter, S. (2003). The development of self-representations during childhood and adolescence. In, M.R. Leary \& J. P. Tangney (Eds.). Handbook of Self and Identity. New York: The Guildford Press.

Jacobson, N. \& Greenley, D. (2001). What is recovery? A conceptual model and explication. Psychiatric Services, 52, 482-485. 
McEvoy, P., Schauman, O., Mansell, W. \& Morris, L. (2012). The experience of recovery from the perspective of people with common mental health problems: findings from a telephone survey. International Journal of Nursing Studies, 49, 1375-1382. DOI:10.1016/j.ijnurstu.2012.06.010

Noiseux, S. \& Ricard, N. (2008). Recovery as perceived by people with schizophrenia, family members and health professionals: A grounded theory. International Journal of Nursing Studies, 45, 1148-1162. DOI:10.1016/j.ijnurstu.2007.07.008

Pilgrim, D. (2008). 'Recovery' and current mental health policy. Chronic Illness, 4, 309310. DOI: $10.1177 / 1742395308097863$

Seginer, R. \& Lilach, E. (2004). How adolescents construct their future: The effect of loneliness on future orientation. Journal of Adolescence, 27, 625-643.

Spaniol, L., Wewiorski, N.J., Gagne, C. \& Anthony, W.A. (2002). The process of recovery from schizophrenia. International Review of Psychiatry, 14, 327 - 336. DOI: $10.1080 / 0954026021000016978$

Steinberg, L., Graham, S., O’Brien, L., Woolard, J., Cauffman, E. \& Banich, M. (2009). Age differences in future orientation and delay discounting. Child Development, 80, $28-44$.

Stickley, T. \& Wright, N. (2010a). The British research evidence for recovery, papers published between 2006 and 2009 (inclusive). Part One: a review of the peer-reviewed literature using a systematic approach. Journal of Psychiatric and Mental Health Nursing, 18, 247-256. DOI: 10.1111/j.1365-2850.2010.01662.x

Stickley, T. \& Wright, N. (2011b). The British research evidence for recovery, papers 
published between 2006 and 2009 (inclusive). Part Two: a review of the grey literature including book chapters and policy documents. Journal of Psychiatric and Mental Health Nursing, 18, 297-307. DOI: 10.1111/j.1365-2850.2010.01663.x

Tew, J., Ramon, S., Slade, M., Bird, V., Melton, J., \& Le Boutillier, C. (2011). Social Factors and Recovery from Mental Health Difficulties: A Review of the Evidence.

British Journal of Social Work, 42, 443-460. DOI:10.1093/bjsw/bcr076

Young, S. L. \& Ensing, D.S. (1999). Exploring recovery from the perspective of people with psychiatric disabilities. Psychiatric Rehabilitation Journal, 22, 219-231. 